\title{
Mathematical concepts and investigative practice
}

\author{
Dirk Schlimm
}

Wednesday, October 5, 2011 (9:01am)

\begin{abstract}
In this paper I investigate two notions of concepts that have played a dominant role in 20th century philosophy of mathematics. According to the first, concepts are definite and fixed; in contrast, according to the second notion concepts are open and subject to modifications. The motivations behind these two incompatible notions and how they can be used to account for conceptual change are presented and discussed. On the basis of historical developments in mathematics I argue that both notions of concepts capture important aspects of mathematical and scientific reasoning, and, consequently, that a pluralistic approach that allows for representing both of these aspects is most useful for an adequate account of investigative practices.
\end{abstract}

\section{Contents}

1 Introduction $\quad 2$

2 Two notions of concepts in philosophy of mathematics 2

2.1 Fregean concepts . . . . . . . . . . . . . . . . . . . . . . 3

2.2 Lakatosian concepts . . . . . . . . . . . . . . . . . 6

3 Mathematical investigations $\quad 8$

3.1 Geometry . . . . . . . . . . . . . . . . . . . . . 9

3.2 Arithmetic and algebra . . . . . . . . . . . . . . . . . 10

4 Notions of concepts and conceptual change $\quad 11$

4.1 Patterns of conceptual change . . . . . . . . . . . . . . . . 11

4.2 Conceptual change: Fregean and Lakatosian . . . . . . . . . . . . . . . . 12

4.3 Towards a pluralistic approach to mathematical concepts . . . . . . . . . . 14

4.4 Notions of scientific concepts . . . . . . . . . . . . . . . . 16

5 Conclusion $\quad 17$ 


\section{Introduction}

One way in which mathematics differs prima facie from empirical science is in its subject matter. Mathematicians do not seem to investigate the empirical world, but rather an abstract, conceptual realm. Consequently, mathematical concepts are not only tools for investigative practice, but are also constitutive of the subject matter itself. In the following section, two notions of mathematical concepts are presented and discussed. According to the first, concepts are definite and fixed; in contrast, according to the second notion they are open and subject to modifications. I will refer to these two notions as 'Fregean' and 'Lakatosian', because they are based on ideas that have been emphasized by Frege and Lakatos respectively and I shall use the writings of these two philosophers to illustrate and motivate them. Nevertheless, it should be kept in mind that these two notions are to a certain extent idealizations and thus are not necessarily those held by Frege and Lakatos themselves. After presenting some historical developments in geometry, arithmetic, and algebra (Section 3), I discuss how such conceptual changes can be accounted for with the use of Fregean and Lakatosian concepts. I argue that both notions capture important aspects of mathematical reasoning and, thus, that an adequate account of investigative practices in mathematics must involve features from each. This insight is not completely novel, as it has already been formulated by the 19th century mathematician Moritz Pasch and others; but philosophers of mathematics, by focusing exclusively on particular mathematical activities (namely proving theorems and creating new mathematics) have been led to consider notions of concepts that are too narrow to account for all aspects of mathematical practice. Finally, I indicate that these considerations are not exclusive to mathematics, but that they also apply to scientific reasoning in general.

\section{Two notions of concepts in philosophy of mathematics}

In this paper I am going to contrast what I regard as the two main notions of concepts that have been put forward and discussed in philosophy of mathematics. Several authors have contributed to these discussions, but the authors who come closest to a clear formulation of these views are Frege and Lakatos, who I turn to next. 


\subsection{Fregean concepts}

Gottlob Frege's tremendous influence on 20th century analytic philosophy is well known. For shaping the mainstream of 20th century philosophy of mathematics his anti-psychologist stance and his views on concepts have been of particular importance. These views are expressed clearly in the introduction to this Grundlagen der Arithmetik (1884):

No, sensations are absolutely no concern of arithmetic. No more are mental pictures, formed from the amalgamated traces of earlier sense-impressions. All these phases of consciousness are characteristically fluctuating and indefinite, in strong contrast to the definiteness and fixity of the concepts and objects of mathematics. (Frege 1884, V-VI; quoted from Frege 1980a, V-VI)

Frege's conception of mathematics as being completely independent of human sensations and mental images, and his explicit demand to always 'separate sharply the psychological from the logical, the subjective from the objective' (Frege 1884, X), kept psychological considerations strictly outside the realm of philosophy of mathematics. Furthermore, Frege promoted a view of mathematics according to which its subject matter is regarded as static, representable by fixed and definite concepts.

Frege's understanding of a concept as being fixed can be interpreted to mean that its extension does not change over time. If an object $a$ falls under a concept $P$ at some point in time, then it always falls under it, eternally. ${ }^{1}$ That a concept is definite means that it is determined for every object, whether it falls under the concept or not. $^{2}$ Concepts that are not definite are also referred to as vague (Black 1937) or fuzzy concepts (Zadeh 1965). Such fixity and definiteness of concepts are necessary for a formal account of valid inferences. If the extension of a concept fluctuated over time, the truth value of universal statements could change during the course of a single proof, thus not allowing for sound rules of inference. Consider the situation where we take as premises 'Pluto orbits around the sun' and 'If Pluto orbits around the sun, then Pluto is a planet'. This licenses us to infer by modus ponens that 'Pluto is a planet'. However, if the concept of planet changed its extension in the time between the formulation of the

\footnotetext{
${ }^{1}$ The extension of certain concepts, like 'being tired,' is certainly relative to a specific time, but Frege is restricting his attention to mathematical concepts, which are not intrinsically tied to space and time; Quine formulates this restriction by referring to eternal sentences, 'whose truth value stays fixed through time and from speaker to speaker' (Quine 1960, 192).

${ }^{2}$ Formally, the notions under consideration are: $P_{S}(a) \leftrightarrow \forall t P_{t}(a)$, the extension of a concept does not change over time, and $\forall t \forall x\left(P_{t}(x) \vee \neg P_{t}(x)\right)$, i. e., at each point in time, either an object falls under $P$ or it does not (for $s$ and $t$ ranging over points in time, an object $a$, and a concept $P$, with $P_{s}(a)$ meaning that the object $a$ falls under concept $P$ at time $s$.)
} 
premises and the statement of the conclusion in such a way that Pluto was no longer a planet, then the assumptions were true, but the conclusion false, i. e., the inference would be invalid! Similarly, the syllogistic inference from 'All swans are black' and 'All black things are pretty' to 'All swans are pretty' would be invalid if the concept of swan changed its meaning between the formulation of the premises and the statement of the conclusion in such a way that it was extended to include an ugly white swan.

A very similar argument was adduced by Poincaré in response to the question regarding the necessary conditions for the application of the rules of logic. ${ }^{3}$ The conclusion he reached was that 'the classification which is adopted be immutable' (Poincaré 1909, 461; quoted from Poincaré 1963, 45). What he means by 'immutable' is that the extension of the concepts remain fixed, as he illustrates with the following example. Since a brigade of soldiers is constituted by regiments, it follows logically that two soldiers who are in the same regiment are also in the same brigade. But, this requires the concepts to remain fixed during the course of an argument:

We learn that two soldiers are members of the same regiment, and we want to conclude that they are members of the same brigade; we have the right do to this provided that during the time spent carrying on our reasoning one of the two men has not been transferred from one regiment to another. (Poincaré 1963, 45; emphasis added by DS)

The definiteness of concepts guarantees the law of the excluded middle, a logical principle formulated by Aristotle and accepted by Frege. Moreover, as Black has argued, concepts that are not definite lead to logical contradictions (Black 1937, 436). Thus, without keeping the extension of concepts fixed and without concepts being definite, no general relationships between them could reliably be expressed once and for all. In Frege's words:

as regards concepts we have a requirement of sharp delimitation; if this were not satisfied it would be impossible to set forth logical laws about them. (Frege 1891, 20, quoted from Beaney 1997, 141; see also Frege 1884, §1)

For Frege, the development of a 'logically perfect system' is the ultimate goal of mathematics, as he explained in a letter to Hilbert (Frege 1980b, 44). To be able to express conceptual relations unambiguously Frege invented the language of predicate logic or, as he called it, the 'concept script' (Begriffsschrift). This new language was intended to overcome the ambiguities and limitations of natural language and to allow

\footnotetext{
${ }^{3}$ I am grateful to Michael Hallett for pointing out this passage to me. For a more detailed discussion of Poincaré's position, see (Hallett 2011, 194-200).
} 
for rigorous, gap-free arguments that could be carried out without any recourse to intuition. The determinateness (encompassing both fixity and definiteness) of concepts was a requirement, indeed the only requirement, for these logical investigations:

All that can be demanded of a concept from the point of view of logic and with an eye to rigour of proof is only that the limits to its application should be sharp, that we should be able to decide definitely about every object whether it falls under that concept or not. (Frege 1884, § 74, quoted from Frege 1980a, 87)

Here Frege introduces a further subtlety regarding the definiteness requirement for concepts. Not only must it be determined which objects fall under a concept and which do not, but we must be able to ascertain which one of these is the case. In the second volume of Grundgesetze der Arithmetik (1903) Frege formulates this requirements somewhat differently as a demand for definitions, and calls it the 'Principle of completeness', the first of his 'Principles of definition':

A definition of a concept (of a possible predicate) must be complete; it must unambiguously determine, as regards any object, whether or not it falls under the concept (whether or not the predicate is truly ascribable to it). Thus there must not be any object as regards which the definition leaves in doubt whether it falls under the concept; though for us human beings, with our defective knowledge, the question may not always be decidable. We may express this metaphorically as follows: the concept must have a sharp boundary. (Frege 1903, § 56, quoted from Beaney 1997, 259; see also p. 298 for a similar formulation)

Now Frege clearly distinguishes between properties of mathematical concepts and our abilities to check whether these properties hold or not. Definiteness is a requirement of concepts, not of our access to them. This understanding of the nature of concepts as being independent of human cognition and epistemological concerns is still widespread in contemporary philosophy of mathematics.

The context of Frege's considerations were the developments in late 19th century mathematics, in particular the emergence of non-Euclidean geometries and Weierstrass' definition of continuous but nowhere differentiable functions, which defied all expectations and intuitions. As a result, mathematicians felt the need to provide some kind of secure foundation for mathematics. This led on the one hand to the axiomatizations of arithmetic by Dedekind and Peano, and on the other hand to Cantor's, Weierstrass', and Dedekind's work on rigorizing the treatment of the continuum. Michael Friedman interprets Frege's general project regarding concepts as being analogous to that regarding mathematics: 
Just as Cantor, Weierstrass, Dedekind, and Peano had finally uncovered the 'true' logical forms of the concepts of infinity and continuity, Frege and his followers could now embark on an analogous project of uncovering the 'true' logical forms of all the concepts found in the mathematical-physical sciences-including, especially, the radically new science of space, time, motion, and matter emerging in the context of Einstein's general theory of relativity. (Friedman 2010, 539)

The followers of Frege are the logical positivist philosophers of the 20th century, who set out to give logical, or rational, reconstructions of concepts, i. e., to give 'an explication of the conceptual contents of scientific concepts ex post facto, as it were, which shows, among other things, how truly rigorous and objective (that is, intersubjective) conceptualization is possible in principle' (Friedman 2010, 539). This program, in particular in the hands of Rudolf Carnap, played a paradigmatic role in 20th century philosophy of science and mathematics.

\subsection{Lakatosian concepts}

A radical break with the static conception, or reconstruction, of mathematics presented in the previous section was put forward by Imre Lakatos in Proofs and Refutations (1976a). Lakatos positions himself squarely in opposition to the logical positivists' aim inherited from Frege - of presenting reconstructions of science and mathematics in formal languages. Instead, he advocates a dynamic view of mathematics based on proofgenerated concepts. Lakatos considers this in opposition to the 'deductivist approach', which 'hides the struggle, hides the adventure' of mathematics (Lakatos 1976a, 142); for him such a conception of mathematics not only covers up the creative work that goes into mathematical investigations, but is also completely inadequate to account for those investigations. After a reference to Carnap's Logische Syntax der Sprache (1934), Lakatos writes: 'Science teaches us not to respect any given conceptual-linguistic framework lest it should turn into a conceptual prison' (Lakatos 1976a, 93, footnote 1).

In support of an alternative view of scientific progress, Lakatos presents - skillfully put into the setting of a classroom discussion - the history of Euler's formula for regular polyhedra, according to which the relation between the number of vertices, edges, and faces is $V-E+F=2$. After starting with the naive conjecture that 'All polyhedra are Eulerian' (i. e., that they satisfy the above formula), various criticisms and counterexamples lead to a series of reformulations or refinements of the conjecture and the involved concepts. Local counterexamples attack particular steps in the argument, while global counterexamples, or 'monsters' in Lakatos' terminology, are 
directed against the conclusion. According to Lakatos' account, in reaction to such couterexamples the statement 'All polyhedra are Eulerian' was initially retained, but the concept of polyhedron acquired a different meaning to exclude the counterexamples. Faced with further objections and challenges the statement was changed to 'All convex polyhedra are Eulerian' and finally to ‘All simple polyhedra with simply-connected faces are Eulerian' (Lakatos 1976a, 41). Of particular importance for the present discussion is the development of the concept of polyhedron, which involved the stretching and contracting of the concept, as well as exploring the relations between what was initially intended by the concept and later definitions that were put forward as responses to criticisms. Understood initially in an informal way the characteristics of the concept of polyhedron were made more and more explicit in reaction to the various proofs and their criticisms. However, Lakatos notes that there is a high price to be paid for this: 'proof-analysis, when increasing certainty, decreases content. [...] Increasing rigour is applied to a decreasing number of polyhedra' (Lakatos 1976a, 57, italics in original). Thus, Lakatos points out a trade-off between conceptual content and rigour, and he is vehemently opposed to a view of mathematics that emphasizes the latter at the cost of the former.

While Lakatos seems to acknowledge in places the importance of sharp mathematical concepts for the purpose of allowing for rigorous deductions, he does not consider this to be of much significance, since he objects to the deductive, 'Euclidean' view of theories. In the following exchange Lakatos even goes so far as to doubt that such sharply defined concepts exist at all:

DELTA: Rationality, after all, depends on inelastic, exact, concepts!

KAPPA: But there are no such concepts! Why not accept that our ability to specify what we mean is nil? If you want mathematics to be meaningful, you must resign of certainty. (Lakatos 1976a, 102, italics in original; Delta and Kappa are students in the imaginary classroom discussion)

That KAPPA here indeed presents a position that is similar to Lakatos' own becomes clear in the footnote to the passage quoted above, in which he objects to the view that ، “clarification" or "explication" of concepts [is] a preliminary to any scientific discussion' (Lakatos 1976a, 90, italics in original). Both components of Frege's determinateness requirement for concepts, namely fixity and definiteness, are thus explicitly rejected by Lakatos, who stresses the importance of the elasticity and inexactness of concepts.

In 'What does a mathematical proof prove?' (1978b) Lakatos elaborates on the relation between proofs and the development of mathematics. Here he distinguishes 
between three kinds of mathematical proof: Pre-formal proofs, which are open ended and not just formal proofs with gaps; formal proofs, which do add rigor to pre-formal proofs, but not much else; and finally, post-formal proofs, which are meta-mathematical results about formalized theories, like the duality principle in projective geometry or the undecidability proofs. Again, Lakatos' arguments are directed against an idealized picture that considers formal theories to be the essence of mathematics. His main claim is that such an approach is unnecessarily restrictive and unable to account for mathematical progress.

While in an informal theory there really are unlimited possibilities for introducing more and more terms, more and more hitherto hidden axioms, more and more hitherto hidden rules in the form of new so-called 'obvious' insights, in a formalized theory imagination is tied down to a poor recursive set of axioms and some scanty rules. (Lakatos 1978b, 160)

Instead of focusing on proofs and the accumulation of timeless truths, Lakatos considers mathematics as driven by finding solutions to problems and thus as being in a state of continual growth and permanent revolution. As such, mathematical practice is much closer to scientific practice than it has been commonly held and its results are fallible 'quasi-empirical' theories. These theories can be falsified by 'heuristic falsifiers,' i. e., informal views about the subject matter in question: 'The crucial role of heuristic refutations is to shift problems to more important ones, to stimulate the development of theoretical frameworks with more content' (Lakatos 1976b, 218). For Lakatos, a view of mathematics that is based solely on Fregean concepts cannot adequately account for these developments.

\section{Mathematical investigations}

In the history of mathematics many concepts were introduced informally and went through considerable changes, redefinitions, splittings, etc. However, other concepts were introduced by formal, determinate definitions from the start. As a consequence, sweeping generalizations regarding the use of concepts in mathematics are difficult to make and, in any case, will most likely fail to account for some aspect of mathematical practice. To get an impression of the diversity of conceptual developments in mathematics, I shall now briefly present some historical episodes. These will be useful in the later discussion of the merits of the different notions of mathematical concepts.

\footnotetext{
${ }^{4}$ Similar arguments against the use of formal and axiomatic theories were put forward by the mathematician Felix Klein (1926, 335-336).
} 


\subsection{Geometry}

Geometric points and lines are mathematical concepts that have been around for well over two thousand years. In Euclid's famous Elements they are introduced as follows:

1. A point is that which has no part.

2. A line is a breadthless length. (Heath 1956, 153)

Their relations are specified by postulates (and common notions), the first one of which reads 'To draw a straight line from any point to any point' (Heath 1956, 154). While Euclid and his fellow Greek geometers were able to derive astonishing consequences from these initial assumptions, e.g., the famous Pythagorean theorem, the nature of the concepts involved and of our epistemic access to them was a matter of much philosophical debate (Mueller 1981). Nonetheless, geometry thrived and bold investigations regarding the dependence relationships among Euclid's axioms eventually led to the emergence of non-Euclidean geometries, i. e., theories in which one of Euclid's postulates, the Parallel Postulate, is replaced by an incompatible one. ${ }^{5}$ While the origin of the concepts of Euclidean geometry can be traced back to empirical observations, the concepts of non-Euclidean geometries were based from the beginning on axiomatic characterizations. In fact, visualizations or intuitive models of these geometries were developed only decades after the theories had been developed to a considerable degree. In the wake of these achievements, mathematicians were suddenly faced with the problem of distinguishing between different kinds of geometric points and lines (Euclidean and non-Euclidean). But that wasn't the end of it. In the 19th century another kind of geometry was also studied in great detail, namely projective geometry, in which a curious relationship (called the 'principle of duality') holds between points and lines: In any theorem of projective geometry, if the terms 'point' and 'line' are interchanged and the relations are changed accordingly, the result is again a theorem of projective geometry. Mathematicians were perplexed about this behavior, since, prima facie, points and lines do not seem to be interchangeable. These developments made it even more difficult to understand the basic concepts of points and lines. At first these concepts had seemed to be very tightly connected to drawn figures, possibly obtained from such figures through a process of abstraction or idealization. But this relation between geometrical concepts and visual figures became thinner and thinner with the emergence of new and mutually incompatible notions of points and lines. In Hilbert's celebrated Grundlagen der Geometrie (1899), the referents of the terms points and lines were finally allowed to be any objects of thought that satisfied the axioms. In other words,

\footnotetext{
${ }^{5}$ See (Bonola 1955) and (Gray 2007) for an account of these developments.
} 
only the relational structure that was characterized implicitly by the axioms was taken to matter for geometry; the concepts of points and lines vanished and all that remained were roles in a system of concepts that define a geometric space. This is a structuralist conception of mathematics (Reck and Price 2000). As a philosophical position it is currently held in high esteem, although it is certainly not accepted by everybody in particular, if connected to a realist view regarding the ontology of mathematical structures; but as a methodological account of contemporary mathematics it seems to be largely uncontroversial. In any case, our focus here is not the metaphysics of mathematics, but the role of concepts in mathematical investigations. It is also worth pointing out that this development of geometry was by no means a smooth one: it took many decades of heated debates before non-Euclidean geometries were accepted as genuine mathematical theories, and Hilbert's account was heavily criticized - most famously by Frege (Hallett 2010).

\subsection{Arithmetic and algebra}

With regard to the basic concepts of arithmetic, like number, zero, integer, etc., a similar story to that of geometric concepts can be told. Again, in Euclid's Elements (Book VII) we find the definition of 'number' as 'a multitude composed of units' and 'A unit is that by virtue of which each of the things that exist is called one' (Heath 1956, 277). Definition 11 introduces prime numbers as 'that which is measured by an unit alone' (Heath 1956, 278). So, according to these definitions the concept of number applies only to $2,3,4, \ldots$, since the unit is not itself a number. The origins of the concept of zero are difficult to trace back, because according to our common conception of it, it is the symbol for an empty place-value in the decimal system (e. g., in '101') as well as the symbol denoting the absence of a magnitude (e.g., in 'the number of round squares is zero'). These notions (and others that are now associated with zero) are, however, independent from each other and, historically, were not always all represented by the same symbol (Schlimm and Skosnik 2011). After the incorporation of zero and one into the number system, the concept of number was gradually, and not without objections, extended further to cover also the negative numbers, fractions, real numbers, and complex numbers. Each of these steps required a reconceptualization that involved giving up some of the properties that had previously been considered essential for numbers. The status of Cayley's octonions and Hamilton's quaternions is still ambiguous: While they are sometimes simply classified as algebras, one can also find them referred to as 'hypercomplex numbers.' 
The concept of integer is sometimes used in an absolute sense to denote the positive and negative whole numbers $(\ldots,-2,-1,0,1,2,3, \ldots)$, but it is also used in algebraic number theory as being relative to a given number field. This is related to investigations in the 19th century that led to a change regarding the defining characteristics of the concept of prime numbers. Within the natural numbers, Euclid's definition stated above is equivalent to the following, second definition: $x$ is prime if, whenever $x$ divides a product $a b$, then $x$ divides either $a$ or $b$. However, in the ring $\mathbb{Z}[\sqrt{5} i]=\{a+b \sqrt{5} i \mid a, b \in \mathbb{Z}\}$ the number 2 is a prime according to Euclid's definition, but not according to this second definition, since 2 divides 6 , but it neither divides $1-\sqrt{5} i$ nor $1+\sqrt{5} i$, which are also factors of 6 . Faced with this dilemma, mathematicians had to decide which property to regard as the definition of primality and they settled for the second one, possibly due to its having greater explanatory power (Tappenden 2008, 268). They renamed the property determined by Euclid's definition 'irreducibility.'

\section{Notions of concepts and conceptual change}

\subsection{Patterns of conceptual change}

Rather than analyzing the particular episodes of conceptual changes from the history of mathematics presented above in more detail, I would like to focus our attention on some general patterns and discuss these in terms of the two notions of concepts introduced in Section 2 above.

A useful starting point for discussing conceptual change is Kitcher's study of patterns of mathematical change in terms of rational transitions between mathematical practices (Kitcher 1983). Such a practice is characterized by Kitcher as consisting of five components: a language, meta-mathematical views (including standards of proof and definition, and claims about the scope and structure of mathematics), a set of questions selected as important, a set of accepted reasonings, and a set of accepted statements. For Kitcher, transitions between practices result from answering questions, generating new questions, generalizing, rigorizing, and systematizing. Answering questions that are deemed relevant by the mathematical community often involves the acceptance of new statements (theorems) and also the introduction of novel forms of reasoning. The study of projective geometry in order to provide a unified treatment of geometric proofs and the extension of the real numbers to the complex numbers are examples of developments that resulted from the desire to answer open questions. New questions 
can be introduced into mathematics from practical considerations in everyday life or other sciences (Wilson 2006), but also from within mathematics itself. In the transfer of questions from one domain to another analogical reasoning plays an important role. When a new theory is developed to go beyond previous ones, Kitcher speaks of generalization (e.g., the introduction of negative numbers to allow for subtraction between any two numbers, and Cantor's extension of arithmetic to transfinite numbers). Rigorization mainly involves a reconsideration of the accepted modes of reasoning, while systematization aims at reorganizing a given body of knowledge. Kitcher distinguishes between systematization by axiomatization (e.g., Euclid's axiomatization or the axiomatizations of group theory) and systematization by conceptualization, where new definitions and questions are applied to some previously developed mathematics (e.g., Lagrange's identification of the form of equations that are amenable to certain techniques of root finding).

In Kitcher's analysis, mathematical concepts are expressed by terms in the given language, so that any change in the language has effects on the concepts involved. Thus, from the historical episodes sketched in the previous section together with reformulations of Kitcher's interpractice transitions in terms of how they effect changes in the underlying language, we can extrapolate a few general patterns of conceptual changes in mathematics: Clarifications of informal concepts, systematizations of concepts and results, investigations of sharp concepts (defined by axiom systems), and generalizations and abstractions. Each of these patterns can result in new or modified mathematical concepts.

\subsection{Conceptual change: Fregean and Lakatosian}

Given the examples from mathematics (Section 3) and the patterns of conceptual changes discussed in Section 4.1 above, we can now ask: Which of the two notions of mathematical concepts, the Fregean or the Lakatosian, is better suited for an adequate account of these changes?

Let us take for granted that at some point in time the term 'number' was used only for the natural numbers greater than one, but that later one and zero were included, and that later still the concept was extended to cover rational, real, and complex numbers as well. According to the Fregean notion of concepts every concept is definite and fixed, i. e., it is clearly determined which objects fall under it and which do not, and the extension of a concept does not change over time. Thus, the concept that includes only the natural numbers greater than one is different from the concept that also includes 
one, which is again different from the concept that includes one and zero. For the sake of clarity, one should thus distinguish between the three concepts number $>1$, number $>0$, and number $\geq 0$. Since concepts are fixed, none of these concepts changed in any way in the course of history; rather, what changed was our usage of the term 'number': while Euclid used the term (or a Greek translation of it) to refer to the concept number $_{>1}$, it later came to refer to the concepts number $>0$ and number $\geq 0$. In fact, these different concepts can still be referred to in the current language of mathematics, e. g., by speaking of 'the positive whole numbers' or 'the positive integers' to refer to number $>0$.

Since the Fregean account does not consider concepts as being relative to the mathematician or mathematical community at any particular time, it allows for a description of mathematics that is independent of any human agents and their contingent properties. Particular mathematicians simply grasp one concept or the other. Under the assumption that mathematics per se corresponds to the current state of mathematics, we can speak of somebody grasping the 'right' concept just in case it corresponds to the currently accepted one. Such a view is flattering to current mathematicians and philosophers of mathematics and it allows for clear identifications of mathematical heroes, namely those who were able to grasp the 'right' concepts first, but it also makes most mathematicians of the past look like they were mistaken most of the time. A more charitable interpretation of the past in terms of Fregean concepts would be one in which the past mathematicians grasped the 'right' concepts from the start, but had difficulties in expressing them properly. From this perspective, Euclid had the concept of prime numbers (the same we use nowadays), but he failed to latch onto the correct defining characteristic since he was misled by the fact that for the natural numbers, the irreducible numbers and prime numbers are co-extensional. Another possible interpretation would be that Euclid grasped a concept that was 'in the vicinity' of our current concept of primality, but that he had difficulties in narrowing in exactly which concept primality was. ${ }^{6}$ The problem of picking the 'right' concept is indeed one that one finds frequently expressed in accounts of mathematical research.

From a Lakatosian perspective on concepts, changing a concept poses no difficulties. With regard to the development of the concept of number mentioned above, it simply changed in such a way that it became more and more inclusive. With regard to the notion of primality, the concept changed its defining characteristics from the Euclidean ones to those now used in modern algebra. Such an account of the history of mathematics in terms of the open notion of concept that was put forward by Lakatos tends to highlight

\footnotetext{
${ }^{6}$ I am grateful to Brian van den Broek for suggesting to me this interpretation.
} 
certain continuities in the development, rather than the discrete changes from one Fregean concept to another.

Closely related to a Lakatosian notion of concepts are evolutionary accounts of conceptual change. The mathematician Raymond Wilder put forward such an account based on the metaphor of mathematics as an organism (Wilder 1953, 438). He presents an organic, evolutionary view of the development of mathematical concepts that are conceived, have a certain life-span, and then die. Wilder emphasizes the mathematical community as the fertile ground in which concepts thrive: Previous concepts are synthesized using available tools and collaborations promote cross-fertilization. His main example, the development of the concept of curve is similar in many respects to Lakatos' account of the history of Euler's theorem for polyhedra. More recently Madeleine Muntersbjorn (2003) has also pointed out the evolutionary character of concept development in mathematics by speaking of the 'cultivation' of concepts.

While historical accounts of the development of mathematics are likely to focus on different issues depending on the notion of concepts that is employed, there does not seem to be any a priori reasons for preferring one to the other. A history of mathematics can be formulated using either Fregean or Lakatosian concepts. However, metaphysical views regarding the nature of mathematics may well influence the notion of concepts that one employs. For example, a Platonist, who thinks that mathematical objects exist in some eternal, acausal realm, will be inclined to analyze historical developments using a Fregean notion of concept, while somebody who links the possession of concepts to sensible experiences and who has no difficulty admitting changes to the concepts themselves might prefer an analysis in terms of Lakatosian concepts.

\subsection{Towards a pluralistic approach to mathematical concepts}

The above discussions brought out different reasons in favor of both a Fregean and a Lakatosian notion of mathematical concepts. On the one hand, keeping with classical conceptions of logical inference requires concepts to be definite and fixed. Moreover, mathematicians themselves do sometimes define their concepts (often understood as higher-order relational concepts, e. g., that of a Euclidean space) by systems of axioms and thereby provide clear-cut conditions for whether something falls under a concept or not. On the other hand, the history of mathematics is full of episodes that appear to be characterized best by an account according to which definitions and extensions of concepts change over time. This also is encouraged by the frequent reuse and adaptation of mathematical terminology. 
Influenced by his analyses of the evolution of the concepts of function, continuity, integrability, series summation, and group, Kitcher summarizes:

Originally the reference of the associated terms was fixed through paradigms. Later discussions show a sequence of attempts to give a descriptive characterization of the entities which had previously been picked out. (Kitcher 1983, 171)

As an example he discusses the development of the concept of function. For Leibniz, functions of a curve were given paradigmatically as its length and area. But the concept was developed further, leading to Euler's 'partial descriptive characterization' of a function as any expression made up of variables and constants, and eventually resulted in the modern set-theoretic characterization (Kitcher 1983, 172). A concept that is introduced via a paradigm is more like a Lakatosian concept, while one that is defined by a descriptive characterization is more like a Fregean one. Thus, Kitcher's characterization can be interpreted as a move from Lakatosian to Fregean concepts in mathematics. Similarly, the general trend of the historical development of mathematics is interpreted by Buldt and Schlimm (2010) as leading from a 'bottom-up' approach to mathematical concepts - based on an Aristotelian understanding of abstraction and resulting in Lakatosian concepts - to a 'top-down' introduction of determinate mathematical structures by means of axiom systems, resulting in Fregean concepts.

It is interesting to note that a related distinction was also made by the 19th century mathematician Moritz Pasch, who was interested both in the dynamic character of mathematics as well as in the need for fixed and determinate mathematical concepts. Unlike the authors mentioned above, Pasch did not draw the line diachronically, but between different mathematical activities. He distinguished between the 'rigid' (starr) part of mathematics, which is governed by the rules of logic, and the 'pliable' (biegsam) part of mathematics, which is open-ended and creative.

In the latter, more libertine, part of geometry and of mathematics generally, all are free to do as they will. In the former part, every move is constrained by the iron laws of deductive logic. I call these the pliable and the rigid parts of mathematics. (Pasch 1920, quoted from Pollard 2010, 100)

For the correct application of deductive reasoning, Pasch is very clear that it must be decidable whether something falls under a concept or not (Pasch 1914, 153-159; see also Schlimm 2010, 105). Thus, in the terminology introduced above, Pasch requires Fregean concepts for rigorous, rigid mathematics in which giving proofs is the main activity. But he acknowledges the usefulness of open, Lakatosian concepts for mathematical reasoning that leads to new discoveries. However, Pasch does not consider the 
development of mathematics to necessarily involve replacing Lakatosian concepts by Fregean ones. Instead, the use of these kinds of concepts can alternate:

I distinguish between the rigid and pliable parts of mathematics. The pliable part includes the 'pre-mathematical' discussions that precede purely mathematical work or are interpolated between purely mathematical contributions. (Pasch 1926, quoted from Pollard 2010, 237; emphasis by DS)

Thus, for Pasch, we do not have to decide between a Fregean and a Lakatosian notion of concepts once and for all, nor is mathematics characterized by a development from Lakatosian to Fregean concepts. Rather, we will understand mathematics better if we consider mathematical practice to alternate between these notions, depending on the context of the investigations. ${ }^{7}$

\subsection{Notions of scientific concepts}

While historians and philosophers of science often have a different understanding of scientific concepts than the two presented above concerning mathematical concepts, it seems that the main point of the previous considerations regarding mathematical concepts also carries over to scientific concepts in general. For in science, too, there is a tension between the need for determinate concepts in reasoning and for open concepts to accommodate conceptual changes.

While logical positivists adhered mainly to a Fregean notion of concepts, the historical turn in philosophy of science with its emphasis on scientific creativity and discovery emphasized a rather different notion. Lakatos' and Kitcher's talk of 'revolutions' and 'paradigms' seen above clearly recall Kuhnian ideas, particularly the distinction between normal and revolutionary science. A Fregean notion of concepts fits well in an account for the more structured developments within normal science, while a Lakatosian understanding might appear better suited for times of fundamental changes. In this vein, Hofstadter explicitly opposes 'Platonic' (Fregean) concepts to 'fluid' (Lakatosian) concepts and argues that the latter are necessary for creative reasoning, e. g., by analogy. For him fluid concepts are 'concepts with flexible boundaries, concepts whose behavior adapts to unanticipated circumstances, concepts that will bend and stretch - but not without limit' (Hofstadter 1995, 307; see also p. 119). The importance of such underspecified or 'open-textured' predicates for analogical reasoning has also been noted recently by Bartha. Whether such a predicate applies is something that needs

\footnotetext{
${ }^{7}$ The observation that concepts are often framed with a particular purpose in mind is also addressed in the studies of Brigand and Steinle, this volume.
} 
to be argued for and that frequently 'involves a nontrivial comparison to paradigm cases or prototypes' (Bartha 2010, 9). However, Bartha's nuanced analysis of analogical reasoning also reveals the tension between fixed and open concepts, and the need for both notions when reasoning and creativity are combined. He writes:

[...] analogical arguments in science depend upon basic similarities that are supposed to be unproblematic. The relevant concepts have a clear meaning over a range of cases including those under discussion; they are not open-textured in the context of that argument. By contrast, it is not uncommon for the conclusion of an analogical argument in science to extend an open-textured predicate to a new case. (Bartha 2010, 10; emphasis in original)

These considerations are directly tied to Lakatos' historical reconstruction discussed in Section 2.2 above, which Bartha analyzes as follows, again invoking both notions of concepts:

Here, too, open-textured concepts are the ones under investigation. They figure in the conclusions of analogical arguments. The starting similarities, however, are independently acceptable and the relevant predicates are not treated as opentextured. (Bartha 2010, 10)

We see here that Bartha's considerations are remarkably similar to those of Pasch, emphasizing the need for both open-textured, Lakatosian concepts and closed, Fregean concepts, depending on the particular context of reasoning.

\section{Conclusion}

Much of mathematical research has to do with introducing new concepts and this is often done on the basis of a paradigm or a not yet fully articulated understanding of their defining characteristics. This insight has been vividly brought to the attention of philosophers by Lakatos. For the purpose of deductive arguments, however, concepts have to be assumed to be definite and fixed, as was pointed out repeatedly, e. g., by Frege, Pasch, and Poincaré. By focusing exclusively on only one of these two mathematical activities one is easily lead to opposing conceptions of mathematical concepts: The notions of closed, Fregean concepts and open, Lakatosian concepts. I have argued that while both can be used for describing the history of mathematics, each of them also captures an important aspect of mathematical practice. Thus, or a more complete and rich understanding of the development of mathematics and science as human activities a pluralistic approach appears to be most fruitful, which allows for both Fregean and 
Lakatosian notions to play a role depending on the particular context in which a concept is used.

Acknowledgements. I would like to thank the organizers and participants of the workshop 'Scientific Concepts and Investigative Practice', held January 7-8, 2011 at Technische Universität Berlin, Germany, as well as Tyler Call, Oran Magal, Michael Hallett, Rachel Rudolph, Brian van den Broek, and Andy Yu for helpful comments on an earlier version of this paper.

\section{References}

Bartha, P. F. A. (2010). By Parallel Reasoning: The Construction and Evaluation of Analogical Arguments. New York: Oxford University Press.

Beaney, M. (Ed.) (1997). The Frege Reader. Blackwell Publishers, Oxford.

Black, M. (1937). Vagueness. an exercise in logical analysis. Philosophy of Science 4(4), 427-455.

Bonola, R. (1955). Non-Euclidean Geometry: A Critical and Historical Study of its Development. New York: Dover.

Buldt, B. and D. Schlimm (2010). Loss of vision: How mathematics turned blind while it learned to see more clearly. In B. Löwe and T. Müller (Eds.), Philosophy of Mathematics: Sociological Aspects and Mathematical Practice, pp. 87-106. London: College Publications.

Carnap, R. (1934). Logische Syntax der Sprache, Volume 8 of Schriften zur wissenschaftlichen Weltauffassung. Vienna: Springer. Revised and expanded English translation published by Kegan Paul Trench, Trubner \& Co., London, 1937.

Frege, G. (1884). Grundlagen der Arithmetik. Verlag von Wilhelm Koeber, Breslau. English translation by J. L. Austin: (Frege 1980a).

Frege, G. (1891). Funktion und Begriff. Jena: Hermann Pohle. Translated, as 'Function and Concept', by Peter Geach; reprinted in (Beaney 1997).

Frege, G. (1903). Grundgesetze der Arithmetik, Volume 2. Jena: Verlag Herrman Pohle. Reprinted by Georg Olms Verlagsbuchhandlung, Hildesheim, 1962. English translation of excerpts by Michael Beaney in (Beaney 1997).

Frege, G. (1980a). The Foundations of Arithmetic: a logico-mathematical enquiry into the concept of number (2nd rev. ed.). Evanston, Ill.: Northwestern University Press. English translation by J. L. Austin.

Frege, G. (1980b). Philosophical and Mathematical Correspondence. University of Chicago Press. Edited by Gottfried Gabriel, Hans Hermes, Friedrich Kambartel, Christian Thiel, and Albert Veraart.

Friedman, M. (2010). Logic, mathematical science, and twentieth century philosophy: Mark Wilson and the analytic tradition. Noûs 44(3), 530-544. 
Gray, J. (2007). Worlds Out of Nothing. A Course in the History of Geometry in the 19th Century. London: Springer.

Hallett, M. (2010). Frege and Hilbert. In M. Potter and T. Ricketts (Eds.), The Cambridge Companion to Frege, pp. 413-464. Cambridge University Press.

Hallett, M. (2011). Absoluteness and the Skolem Paradox. In D. DeVidi, M. Hallett, and P. Clark (Eds.), Logic, Mathematics, Philosophy: Vintage Enthusiasms, Number 75 in The Western Ontario Series in Philosophy of Science, pp. 189-218. New York: Springer.

Hallett, M. and U. Majer (Eds.) (2004). David Hilbert's Lectures on the Foundations of Geometry 1891-1902. Berlin, Heidelberg, New York: Springer.

Heath, T. L. (Ed.) (1956). The Thirteen Books of Euclid's Elements (second ed.), Volume 1: Introduction and Books I, II. Dover Publications. Translated from the text of Heiberg with introduction and commentary by Sir Thomas L. Heath.

Hilbert, D. (1899). Grundlagen der Geometrie. Teubner, Leipzig. Reprinted in (Hallett and Majer 2004), 436-525. English The Foundations of Geometry by E. J. Townsend, Open Court, Chicago, 1902 and by L. Under, Open Court, La Salle, Ill., 1971.

Hofstadter, D. R. (1995). Fluid Concepts \& Creative Analogies: Computer models of the fundamental mechanisms of thought. New York: Basic Books.

Kitcher, P. (1983). The Nature of Mathematical Knowledge. Oxford University Press, Oxford.

Klein, F. (1926). Vorlesungen über die Entwicklung der Mathematik im 19. Jahrhundert, Volume 1. Springer Verlag, Berlin.

Lakatos, I. (1976a). Proofs and Refutations. Cambridge University Press, Cambridge. Edited by John Worrall and Elie Zahar.

Lakatos, I. (1976b, Sep.). A renaissance of empiricism in the recent philosophy of mathematics. British Journal for the Philosophy of Science 27(3), 201-223.

Lakatos, I. (1978a). Mathematics, Science and Epistemology, Volume 2 of Philosophical Papers. Cambridge University Press. Edited by John Worrall and Gregory Currie.

Lakatos, I. (1978b). What does a mathematical proof prove? In (Lakatos 1978a), Chapter 4, pp. 61-69. Reprinted in (Tymoczko 1998, 153-162).

Mueller, I. (1981). Philosophy of mathematics and deductive structure in Euclid's Elements. Cambridge, Mass.: MIT Press.

Muntersbjorn, M. (2003). Representational innovation and mathematical ontology. Synthese 134, 159-180.

Pasch, M. (1914). Veränderliche und Funktion. Leipzig and Berlin: B. G. Teubner.

Pasch, M. (1920). Die Begründung der Mathematik und die implizite Definition. Ein Zusammenhang mit der Lehre vom Als-Ob. Annalen der Philosophie 2(2), 144-162. 
Pasch, M. (1926). Die axiomatische Methode in der neueren Mathematik. Annalen der Philosophie 5, 241-274.

Poincaré, H. (1909). La logique de l'infini. Revue de métaphysique et de morale 17, 462-482. Reprinted in Dernières Pensées, Ernest Flammarion, 1913, pp. 7-13; translated in (Poincaré 1963, 45-74).

Poincaré, H. (1963). Mathematics and Science: Last Essays. New York: Dover Publications. Original in French, Dernières Pensées, Ernest Flammarion, 1913. Translated by John W. Bolduc.

Pollard, S. (Ed.) (2010). Essays on the Foundations of Mathematics by Moritz Pasch, Volume 83 of The Western Ontario Series in Philosophy of Science. New York: Springer.

Quine, W. (1960). Word and Object. Cambridge, MA: MIT Press.

Reck, E. H. and M. P. Price (2000, Dec.). Structures and structuralism in contemporary philosophy of mathematics. Synthese 125, 341-383.

Schlimm, D. (2010). Pasch's philosophy of mathematics. Review of Symbolic Logic 3(1), 93-118.

Schlimm, D. and K. Skosnik (2011). Symbols for nothing: Different symbolic roles of zero and their gradual emergence in Mesopotamia. In A. Cupillari (Ed.), Proceedings of the 2010 Meeting of the Canadian Society for History and Philosophy of Mathematics, May 29-31, Montreal, Volume 23, pp. 257-266.

Tappenden, J. (2008). Mathematical concepts and definitions. In P. Mancosu (Ed.), The Philosophy of Mathematical Practice, Chapter 9, pp. 256-275. Oxford University Press.

Tymoczko, T. (Ed.) (1998). New Directions in the Philosophy of Mathematics (Revised and expanded ed.). Princeton University Press, Princeton, NJ.

Wilder, R. L. (1953). The origin and growth of mathematical concepts. Bulletin of the American Mathematical Society 59, 423-448. Reprinted in: Mathematics: People, Problems, Results, Douglas M. Campbell and John C. Higgins (eds.), Wadsworth Int., Belmont, CA, 1984. pp. 239254.

Wilson, M. (2006). Wandering Significance: An Essay on Conceptual Behavior. New York: Oxford University Press.

Zadeh, L. A. (1965). Fuzzy sets. Information and Control 8, 338-353. 